Check for updates

Cite this: RSC Adv., 2018, 8, 16726

Received 20th December 2017

Accepted 27th April 2018

DOI: $10.1039 / c 7 r a 13489 d$

rsc.li/rsc-advances

\section{Binder-free Sn-Si heterostructure films for high capacity Li-ion batteries}

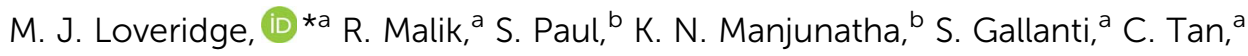 \\ M. Lain, ${ }^{a}$ A. J. Roberts ${ }^{a}$ and R. Bhagat ${ }^{a}$
}

This study fabricated and demonstrated a functional, stable electrode structure for a high capacity Li-ion battery (LIB) anode. Effective performance is assessed in terms of reversible lithiation for a significant number of charge-discharge cycles to $80 \%$ of initial capacity. The materials selected for this study are silicon and tin and are co-deposited using an advanced manufacturing technique (plasma-enhanced chemical vapour deposition), shown to be a scalable process that can facilitate film growth on 3D substrates. Uniform and hybrid crystalline-amorphous Si nanowire (SiNW) growth is achieved via a vapour-liquid-solid mechanism using a Sn metal catalyst. SiNWs of less than $300 \mathrm{~nm}$ diameter are known to be less susceptible to fracture and when grown this way have direct electrical conductivity to the current collector, with sufficient room for expansion. Electrochemical characterisation shows stable cycling at capacities of $1400 \mathrm{~mA} \mathrm{~h} \mathrm{~g} \mathrm{~g}^{-1}(>4 \times$ the capacity limit of graphite). This hybrid system demonstrates promising electrochemical performance, can be grown at large scale and has also been successfully grown on flexible carbon paper current collectors. These findings will have impact on the development of flexible batteries and wearable energy storage.

\section{Introduction}

The US Dept. of Energy cost target for all electric vehicles is \$125 per kWh of usable energy but the current cost of commercial batteries is $\$ 400-500$ per $\mathrm{kWh}^{1}{ }^{1}$ Cost reductions are possible through evolution away from conventional electrode fabrication practices to alternative battery manufacturing schemes. ${ }^{2-4}$ This focuses on processes that do not require multi-stage dispersion, indirect materials (solvents that do not end up in the product), other chemical processes, drying and solvent recovery. At the same time the energy storage community remains heavily engaged in trying to better understand the implications of electron and ion transport within electrode architectures and their influence on electrochemical performance - this is made challenging when there is little control over the electrode structure. ${ }^{5}$ With a better understanding, it is possible to design efficient battery components to overcome such transport issues. Development of electrodes that can retain their microstructure as they are charged and discharged over thousands of cycles is a critical element in creating batteries that will overcome range anxiety in electrified vehicles. ${ }^{6}$

Non-conventional manufacturing methods have been explored to create battery electrodes ${ }^{1}$ yet none of them have inspired commercial uptake into mainstream manufacturing

${ }^{a} W M G$, Warwick University, Coventry, CV4 7AL, UK. E-mail: M.Loveridge@warwick. ac.uk

${ }^{b}$ EMTERC, De Montfort University, Leicester, LE19BH, UK methodology. These include: (1) solvent-based electrostatic spray deposition, ${ }^{7}$ (2) spray painting/electrostatic spraying, (3) dry electrode manufacturing e.g. pulsed laser and sputter deposition, ${ }^{8}$ (4) magnetron sputtering. ${ }^{9}$ Better understanding of the process itself has added valuable insight into directly improving the process for fabricating Li-ion battery electrodes, especially the means by which materials are uniformly deposited. ${ }^{\mathbf{1 0}}$

Intense and widespread research efforts into new materials for Li-ion batteries (LIBs) have focused a lot of interest on the group IV elements ( $\mathrm{Si}$ and $\mathrm{Sn}$ primarily). ${ }^{\mathbf{1 1 , 1 2}} \mathrm{Si}$ and $\mathrm{Sn}$ are the two most studied materials both individually and as an alloy negative electrode materials and both have made some progress towards entering the commercial arena. Cost analysis for these materials shows $\mathrm{Sn}$ be clearly the more economical on a unit cost per Ah basis (graphite $: \mathrm{Si}: \mathrm{Sn}=0.81: 0.08: 1.51 \mathrm{c}$ per Ah) with Si being the second most abundant element on earth. These elements have high lithiation capacities of 3579 and $994 \mathrm{~mA} \mathrm{~h} \mathrm{~g}{ }^{-1}$ respectively, compared with mature graphite anodes of limited capacity of $372 \mathrm{~mA} \mathrm{~h} \mathrm{~g}{ }^{-1} \cdot{ }^{11}$ However, the group IV metals and metalloids interact with Li ions differently $^{\mathbf{1 3}}$ (compared with graphite) and instead of intercalating, they alloy with $\mathrm{Li}^{+}$to the extent of incurring a deleterious volume expansion upon reversible cycling reactions. ${ }^{\mathbf{1 4}, 15}$ This volume increase can reach up to $300 \%$ when approaching the maximum capacity of $\mathrm{Si}$ and such expansion in turn leads to particle pulverisation (Fig. 1), electrode cracking and the progressive growth of a solid-electrolyte interface (SEI). ${ }^{\mathbf{1 6 - 1 8}}$ 


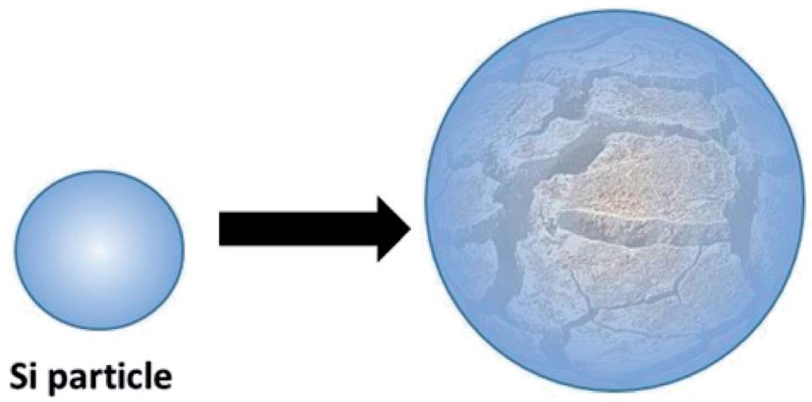

Fully lithiated Si particle

Fig. 1 Illustration of lithiation-induced Si particle fracture.

In contrast to the cathode electrolyte interface (CEI), as is common with materials such as lithium iron phosphate, and the stable SEI formed on graphite, with silicon the SEI is unstable ${ }^{19}$ and continues to grow. ${ }^{20}$ This represents a cumulative, irreversible loss of the Li "inventory" as well as a progressive increase in surface resistance on the Si particles, which results in pronounced and premature capacity decrease as a function of cycle number. This has precluded anodes based on Si from serious attempts at incorporating such materials into batteries for vehicle electrification. There have been an extensive number of studies on both $\mathrm{Si}$ and $\mathrm{Sn}$ as separate active materials for Li-ion batteries ${ }^{21-24}$ but it is not the purpose of this article to incorporate an extensive review of these studies - here we focus on the hybridisation of these elements in a nonconventional manufacturing approach.

More recently there have been attempts at hybridising $\mathrm{Si}$ and Sn within the same electrode, e.g. an investigation using Sn nanoparticles as an effective, conductive additive for Si-based anodes in Li-ion half-cells. ${ }^{25-27}$ The first study claimed that the presence of the Sn (as low as 2\%) dramatically improves the electrode's performance in terms of both charge capacity and cycling stability ${ }^{26}$ It proposed to have achieved this by being uniformly dispersed in the Si network but also reducing the electrical resistance of the electrode structure as a whole. As such, Mangolini et al. the synergistic effects between the materials lead to batteries that exceed the performance of each of the two components alone. ${ }^{25}$ This is attributed to the high electrical conductivity and good reversibly energy storage capacity of Sn. Other researchers have looked at silicon-tin hybrid anode systems for solid-state Li-ion batteries achieving reversible capacities up to $700 \mathrm{~mA} \mathrm{~h} \mathrm{~g}{ }^{-1} \cdot{ }^{28}$ Combining hybridised "yin-yang" silicon-tin porous nanocomposites with graphene has also been used as an approach for generating lowcost and low energy consumption materials with promising electrochemical performance. ${ }^{29}$

When considering silicon as the predominant anode active material, the one-dimensional aspect of silicon nanowires (SiNWs) has received widespread attention ${ }^{30,31}$ but will not be discussed at length here. It reported to be structurally beneficial as an anode active material as it: (1) allows sufficient space between collections of nanowires to accommodate the volume changes brought about by lithiation, and (2) allows axial/radial stress relaxation of the nanowires. ${ }^{32}$ This relaxation is thought to alleviate any progressive pulverisation that is commonly observed in the bulk and thick film Si structures during operational cycling. ${ }^{33,34}$ Metal-assisted vapour-liquid-solid (VLS) mechanism is a widely used approach to obtain anisotropic 1D nanowires owing to its simplicity and versatility with regards to semiconductor nanowires. ${ }^{35,36}$ This method represents an alternative manufacturing route beyond conventional composite electrode fabrication methodology, and could constitute an economically viable, less energy-intense production route within energy storage manufacturing. ${ }^{37}$

The crystalline properties of nanowires grown this way is considered to be of good quality and sometimes referred to as "defect-free", with the exception of multiple twin defects that can cut across the nanowires (described further on in this paper). ${ }^{35} \mathrm{Si}$ is a commonly used alloying element in several grades of aluminium and steel ${ }^{38}$ but does not alloy to a high degree with $\mathrm{Sn}$ since the solubility of Sn in Si is very low $(\approx 5 \times$ $\left.10^{19} \mathrm{~cm}^{-3}\right)^{39}$ as illustrated by the binary phase diagram for the silicon-tin alloy system (see Fig. 2).

Consistent with the Hume-Rothery limits of binary solubility, Sn and Si have atomic radii differences significantly $>15 \%$ and as a result have limited solubility in each other. This is despite their other favourable common properties in this respect, namely crystal structure, valence and electronegativity. ${ }^{41}$ It is this limited solubility with $\mathrm{Si}$ that makes $\mathrm{Sn}$ a good catalyst in this instance as it tends to generate atomically sharp heterostructures. ${ }^{42}$ With this in mind, this study essentially integrates a Sn granular thin film with nano-crystalline and amorphous phase Si nanowires (SiNWs), to generate a degradation-resistant, high capacity anode system. In order to synthesise the $\mathrm{Si}$ nanowires use of Radio-Frequency Plasma Enhanced Chemical Vapour Deposition (RF-PECVD) technique for the nanowire growth that incorporates vapour-liquid-solid (VLS) catalyst mediated growth process, which is described as a "bottom-up" method. ${ }^{43}$ In this process, the substrate coated with Sn as a catalyst is heated in the presence of a hydrogen and silane precursor gas, which preferentially absorbs $\mathrm{Si}$ atoms and precipitated out of the Sn catalyst. Upon dissolution into the Sn droplet, $\mathrm{Si}$ atoms form a liquid eutectic alloy with the underlying $\mathrm{Sn}$ catalyst. Eventually - with continuous flow of $\mathrm{SiH}_{4}$ - the alloy becomes supersaturated whereby the nucleation barrier is surpassed and $\mathrm{Si}$ precipitates at the liquid-solid interface, minimising the free energy of the system. ${ }^{44}$ Because the mechanism consists of adsorption, dissolution, diffusion and precipitation in the liquid phase - these are thermodynamic processes that work towards equilibrium - one can refer to the Sn-Si equilibrium phase diagram in Fig. 2 to understand catalyst mediated growth of solid SiNWs from a liquid catalyst.

As illustrated in the schematic in Fig. 3, the kinetics of the VLS mechanism consist of four major steps:

(1) Mass transport of precursor in the gas phase.

(2) Chemical reactions at the vapour-liquid interface.

(3) Diffusion in the liquid phase.

(4) Incorporation and arrangement of atoms in a crystal lattice. $^{37}$

Whilst studies on VLS SiNW growth are established using Sn as a catalyst, ${ }^{45}$ the application of such films as anode systems for 


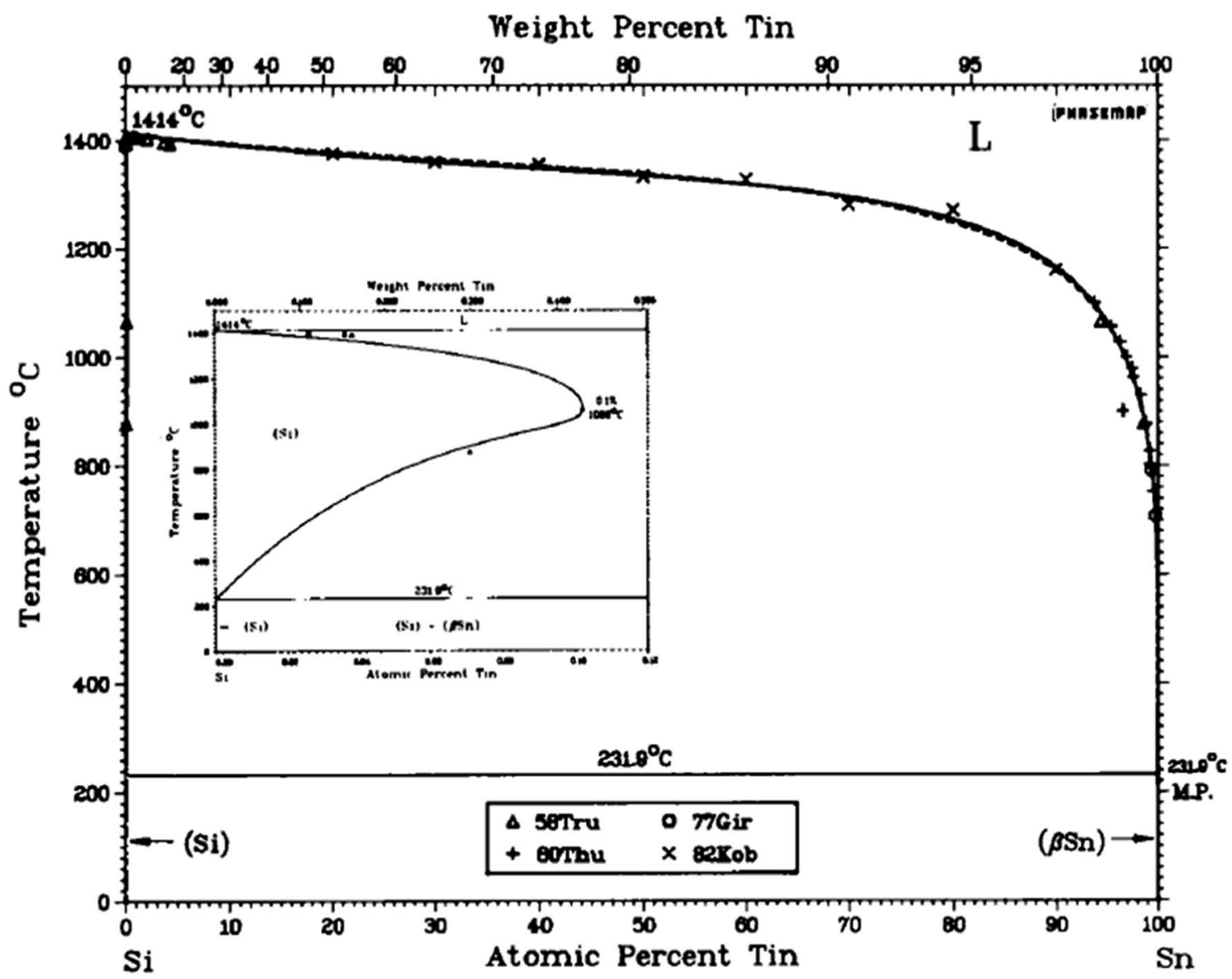

Fig. 2 The $\mathrm{Si}-\mathrm{Sn}$ equilibrium phase diagram ${ }^{40}$.

Li-ion energy storage has not been comprehensively explored. The feasibility of growing these hybrid systems on alternative current collectors is tested here using flexible conductive carbon paper mesh as an advanced 3D electrode manufacturing approach. Flexible free-standing Li-ion batteries have been fabricated using carbon paper (with enhanced conductivity) as the current collector, specifically N-doped 3D porous carbon paper. ${ }^{46}$

\section{Methods/experimental}

a. Plasma enhanced chemical vapour deposition \& vapourliquid-solid SiNW growth

Copper $(\mathrm{Cu})$ foil and $\mathrm{Cu}$ mesh sheets were obtained from Dexmet Corporation and were cut to $30 \times 30 \mathrm{~mm}$ and cleaned with a stream of nitrogen gas. Carbon paper Spectracarb 2050A-0850 was obtained from Toray through-plane resistivity of $18 \mathrm{~m} \Omega$ $\mathrm{cm}^{2}$. Corning glass (Alkali Free Borosilicate-7059) and P-Silicon wafer (500 $\mu \mathrm{m}$ thickness, $1-20 \mathrm{ohm} \mathrm{cm}, 100$ orientation) were cleaned using an RCA process and the native oxide on Si wafers was removed by immersing wafers in a buffered hydrofluoric (HF) acid solution for 10 seconds. The residual HF was removed by rinsing thoroughly with deionised water. The aforementioned substrates ( $\mathrm{Cu}$ foil, $\mathrm{Cu}$ mesh, glass, carbon paper and $\mathrm{Si}$ wafer) were loaded in a thermal evaporator (Edwards AUTO 306) for deposition of $100 \mathrm{~nm}$ (mass thickness) thin film of tin (99.999\% purity) at $10 \AA \mathrm{s} \mathrm{s}^{-1}$ at $8 \times 10^{-7} \mathrm{mbar}$ base pressure.

Real-time thicknesses were monitored using a quartz microbalance. All substrates coated with tin on one side of the substrates were loaded into a capacitive-coupled RF-PECVD (Radio Frequency-Plasma Enhanced Chemical Vapour Deposition) chamber (Oxford PlasmaLab) and pumped down to 5 mtorr base pressure. The temperature was raised to $400{ }^{\circ} \mathrm{C}$ and maintained for $5 \mathrm{~min}$ followed by hydrogen plasma pretreatment (hydrogen gas flow at $100 \mathrm{sccm}, 500$ mtorr chamber pressure and, $33 \mathrm{~mW} \mathrm{~cm}{ }^{-2} \mathrm{RF}$ power density) for $5 \mathrm{~min}$. Without breaking the vacuum, $20 \mathrm{sccm}$ of $\mathrm{SiH}_{4}$ gas was introduced to existing $\mathrm{H}$-plasma to initiate the growth process and growth of silicon nanowires (SiNWs) continued for $15 \mathrm{~min}$. Samples were removed after PECVD chamber was let to cool down to below $50{ }^{\circ} \mathrm{C}$.

\section{b. X-ray diffraction analysis}

In this work, Bruker-D2 phaser equipped with 1-D LYNXEYE detector with a resolution of $\pm 0.02^{\circ}$ is utilised for the investigation. All scans were performed by $\mathrm{Cu}$ anode to produce $\mathrm{X}$-rays at $30 \mathrm{kV}$ and $10 \mathrm{~mA}$ to generate monochromatic X-rays with $1.54056 \AA$ wavelengths. Ni filter is used to remove $\mathrm{K}_{\text {beta }} \mathrm{X}$-rays, thereby only $\mathrm{K}_{\mathrm{alpha}}$ is incident over the sample. SiNWs grown 


.
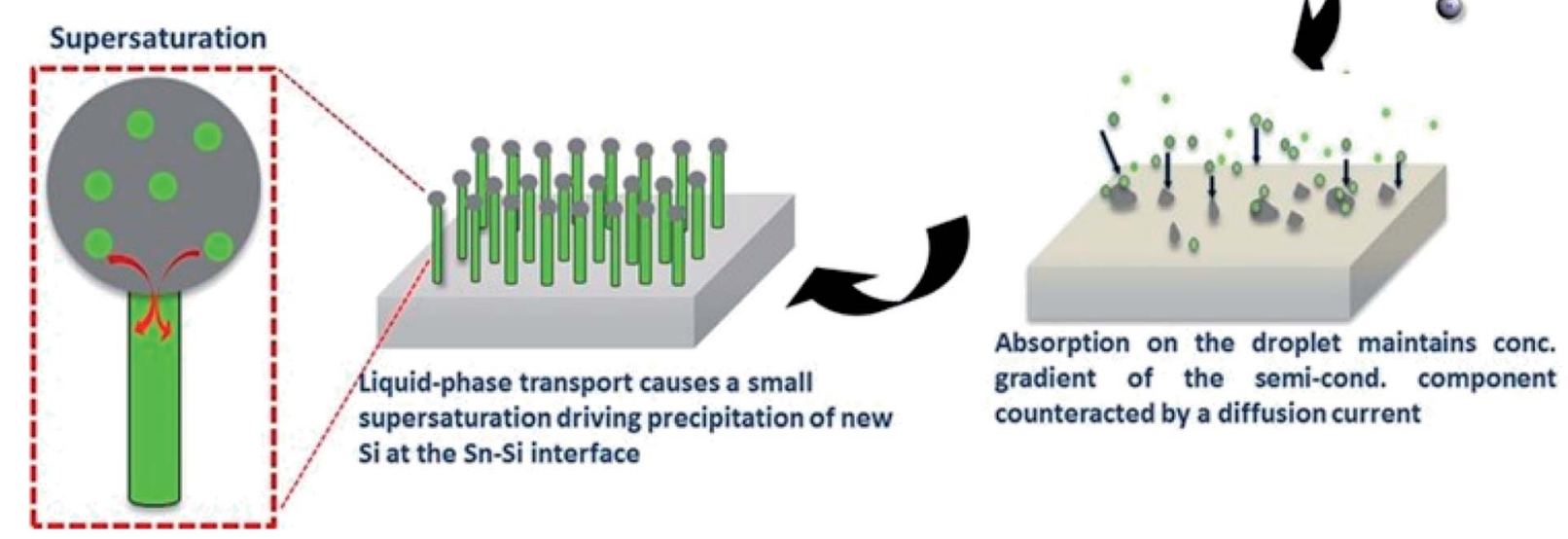

Fig. 3 Schematic illustration of the growth of SiNWs via vapour-liquid-solid mechanism. Sn film coated substrate is exposed to H-plasma to obtain self-assembled Sn spherical nanoparticles. Si atoms are adsorbed and precipitated from Sn nanoparticles to obtain nanowires that are directly grown from the substrate (i.e. nanowires are electrically welded to the bottom conductive Cu sheet or any chosen substrate).

on glass substrates from a tin catalyst with mass thickness $100 \mathrm{~nm}$ is used for investigating structural signature/phase identification.

\section{c. Electrochemical characterisation}

All films were tested in Hohsen 2032 coin cells vs. a lithium foil counter electrode. The separator is $\mathrm{PP} / \mathrm{PE} / \mathrm{PP}$ microporous trilayer membrane (Celgard 2325) and the electrolyte is RD265 (PuriEL, SoulBrain, US) and is composed of EC, EMC, FEC and VC. The cell was cycled galvanostatically using a BioLogic VMP3 potentiostat with a low current module (10 nA limit). Comparison electrodes based on powder $\mathrm{Si}$ and Sn hybrids were fabricated using $50: 50$ active mass ratios (Elkem BV and Sigma Aldrich respectively) in mass proportions of $70: 16: 10$ of active material : polyacrylic acid $(450 \mathrm{k}$ Sigma Aldrich) : Super-P (Timcal). Both cells used the same electrolyte solvent and charge-discharge cycling parameters.

\section{d. Scanning electron microscopy and focused ion beam cross-section preparation}

SEM images were obtained using a Carl Zeiss Sigma Ultra microscope using a working distance of 2-7 $\mathrm{mm}$ and accelerating voltage of $2 \mathrm{kV}$. Cross-section analysis was performed using a $1 \mathrm{nA}$ current at $30 \mathrm{kV}$. For the cross-section analysis Pt was deposited (Fig. 4) to mitigate beam damage to the films. Different currents were used to dig the trench, ranging from 50 , then 30,7 and final finishing with a $1 \mathrm{nA}$ current, all at $30 \mathrm{kV}$ accelerating voltage.

\section{Results and discussion}

The morphology and vertical cross-section microstructure of the vapour deposited Sn-SiNWs film is shown in Fig. 5(a) and (b). The Pt layer seen in the cross-section Fig. 5(b) is used to protect the sample from any damage induced by the ion beam. Identification of the phase composition of the intermetallic $\mathrm{Cu}-$ Sn layer by X-ray diffraction was challenging due to the thickness of the films making detection of the intermetallics very difficult. The Sn catalyst layer in Fig. 5(c) deposited prior to any $\mathrm{Si}$ alloying and precipitation, can be seen to appear as quite a uniformly self-assembled series of spherical deposits that are crystalline. The catalyst is subsequently treated under a $\mathrm{H}$ plasma before the growth of SiNWs following introduction of the silane gas.

The assembly of layered thin films relies on interfacial integrity has been found to have a profound effect on the resulting function. ${ }^{47}$ The formation of intermetallics between $\mathrm{Si}$,

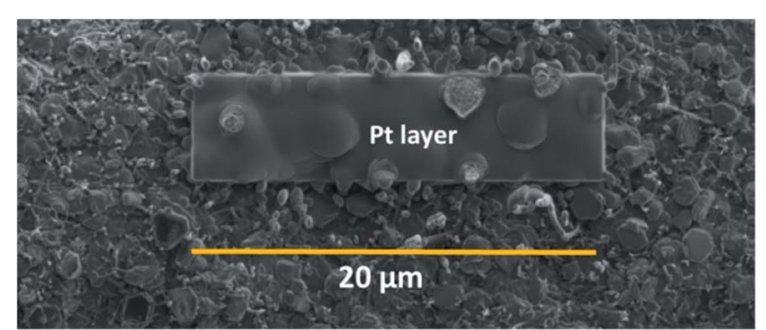

Fig. 4 Sputtered coated Pt over the surface of SiNW-Sn film section for FIB-sectioning. 

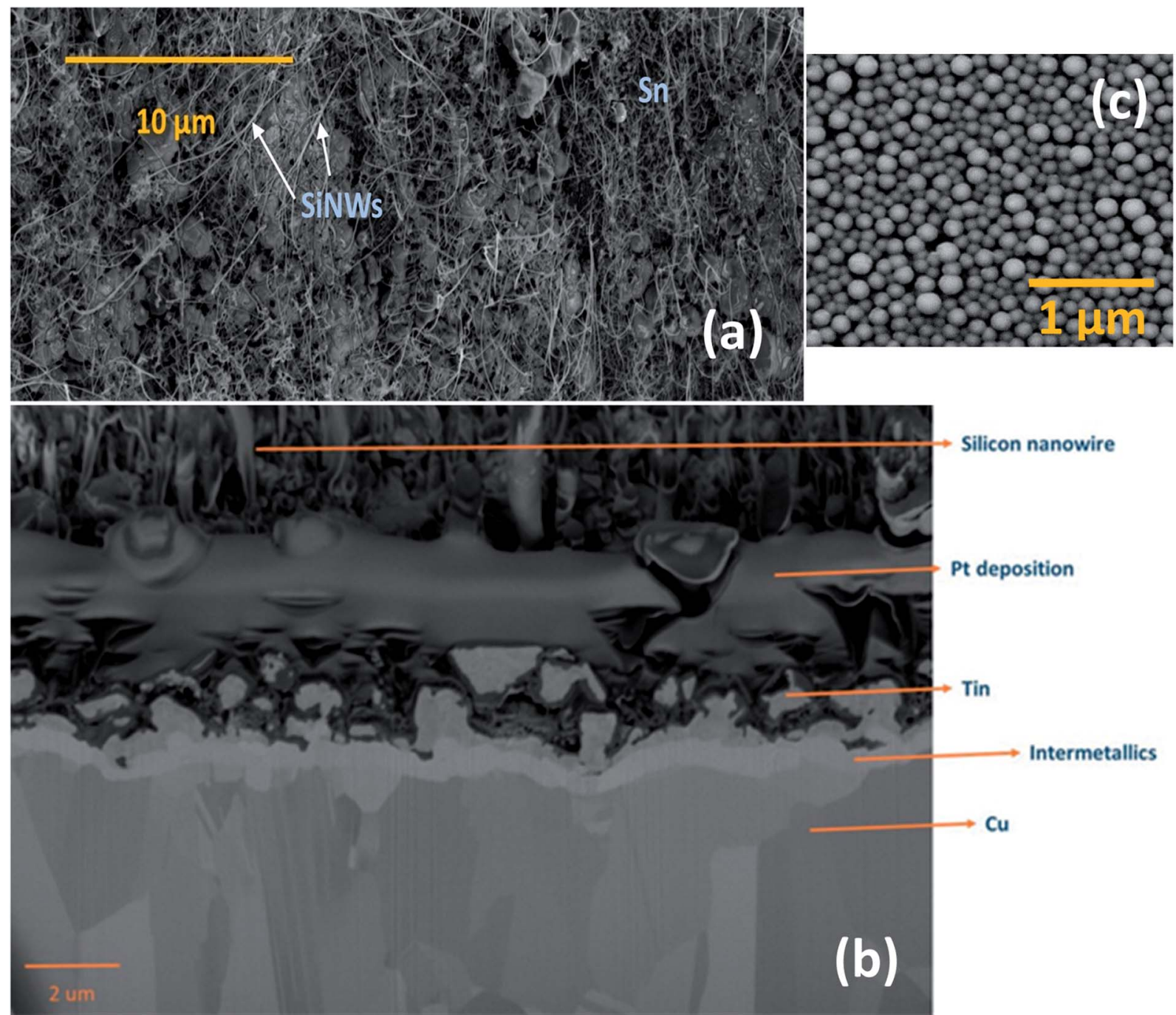

Fig. 5 (a) Morphology of vapour deposited Sn-SiNWs film (top-view). (b) FIB cross-section of SiNW-Sn hybrid film deposited over copper foil. (c) Self-assembled spherical Sn catalyst nanoparticles layer.

$\mathrm{Sn}$ and the underlying $\mathrm{Cu}$ substrate may significantly affect the stability of the interface - this could promote adhesion quality of the active anode film and warrants deeper investigation beyond the experimental scope of this investigation.

The SiNWs have a very high aspect ratio, as can be seen in Fig. 6(a), with diameters typically around $100-150 \mathrm{~nm}$ and lengths of up to $10 \mu \mathrm{m}$, incorporating a significant mass of $\mathrm{SiO}_{2}$ shell. This cannot be directly or very easily quantified as the nanowires are combined with a Sn layer that will have its own associated oxide layer. With higher magnification it is evident that many of the NWs have kinks in their $2 \mathrm{D}$ morphology as shown in Fig. 6(a). The zig-zag morphology found in some of the nanowires is due to stacking-fault energy of $\operatorname{Si}\left(50 \mathrm{~mJ} \mathrm{~m}^{-2}\right)$ - this generates twins during crystallisation ${ }^{35}$ as shown in Fig. 6(b). Twin defects are a special type of grain boundary occurring commonly in many different minerals and are perpendicular to the growth direction of the nanowire. Likely crystalline parameters are illustrated: single crystalline wires are connected by a $120^{\circ}$ angle joint.
Two $\langle 112\rangle_{\mathrm{c}}$ or $\langle 110\rangle_{\mathrm{c}}$ vectors in a cubic crystal structure and two $\langle 11-20\rangle_{\mathrm{h}}$ or $\langle 1-100\rangle_{\mathrm{h}}$ vectors in a hexagonal structure can form such a $120^{\circ}$ joint when rotating about the $\langle 111\rangle_{\mathrm{c}}$ and $\langle 0001\rangle_{\mathrm{h}}$ zone axes respectively. ${ }^{48}$ From the selected area electron diffraction (SAED) patterns in Fig. 7(a) and (b) there is evidence of both crystalline and amorphous phases within the hybrid films deposited in this work. This will have implications in the lithiation behaviour of the anode active materials during charging, this being attributable to charge transfer kinetics in amorphous - compared with crystalline - silicon. ${ }^{49}$ The complex two-phase lithiation of crystalline $\mathrm{Si}$, subsequent lithiation phases that evolve in amorphous $\mathrm{Si}^{50}$ and the recrystallization of $\mathrm{Si}^{51}$ has received much attention and are still yet some way from being definitively concluded.

The crystallinity of these films are further analysed by XRD as shown in Fig. 8. Characteristic peaks that correspond to diffraction planes of Si (blue) and Sn (red) are identified. The diffraction patterns of the deposited composite is purely composed of elemental state of $\mathrm{Si}$ and $\mathrm{Sn}$. Similar patterns 

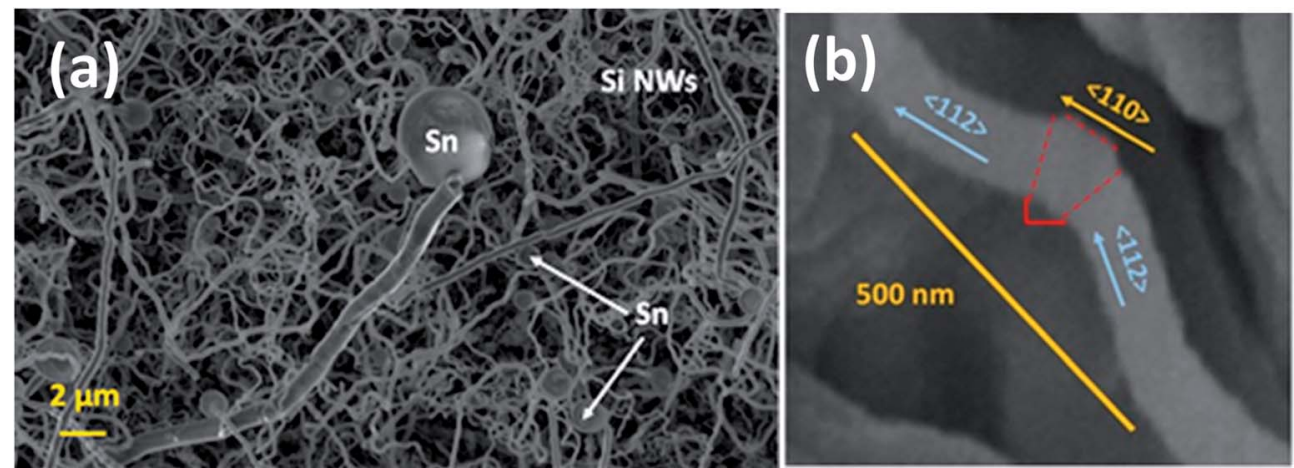

Fig. 6 SEM image of Si-Sn showing (a) the Sn catalyst particles at the tip of SiNWs grown on a copper foil substrate and (b) single SiNW with angular kinks with crystalline phase identification of twinning regions.

corresponding to pure elements have been observed for Si-Sn composites. ${ }^{52}$ The diffraction peak of $\mathrm{Si}$ indicates the cubic phase of SiNWs. These nanowires grown with tin at temperatures used here show increased lattice constants that is attributed to the bending of SiNWs, kinks in the nanowire and incorporation of $\mathrm{Sn}$ in the nanowire during the low temperature growth process. The lattice constant of tin is approximately $20 \%$ larger than silicon..$^{53}$ The tetragonal Sn phase structure is evidenced by the peaks appearing at: $30.6^{\circ}, 32.0^{\circ}, 43.9^{\circ}, 55.3^{\circ}$, $62.5^{\circ}$ and $79.5^{\circ}$ (JCPDS card no. 04-0673).

Fig. 9(a) shows the $1^{\text {st }}$ cycle (formation) for the lithiation and delithiation to $1 \mathrm{~V}$. Five different regions of varying slope can be
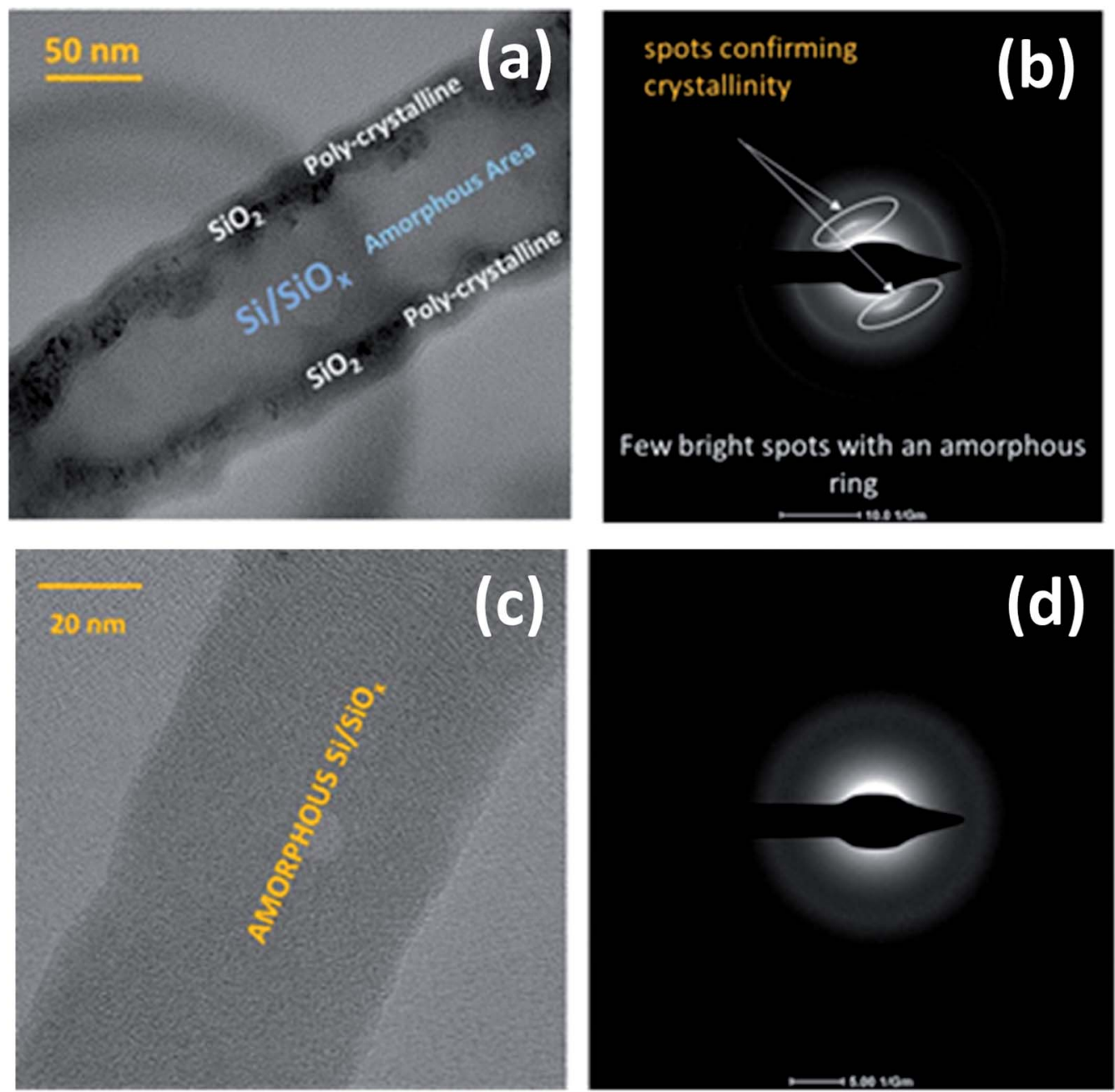

Fig. 7 HRTEM and selected area electron diffraction imaging of SiNW with both crystalline and amorphous regions (a and b) and purely amorphous SiNW (c and d). 


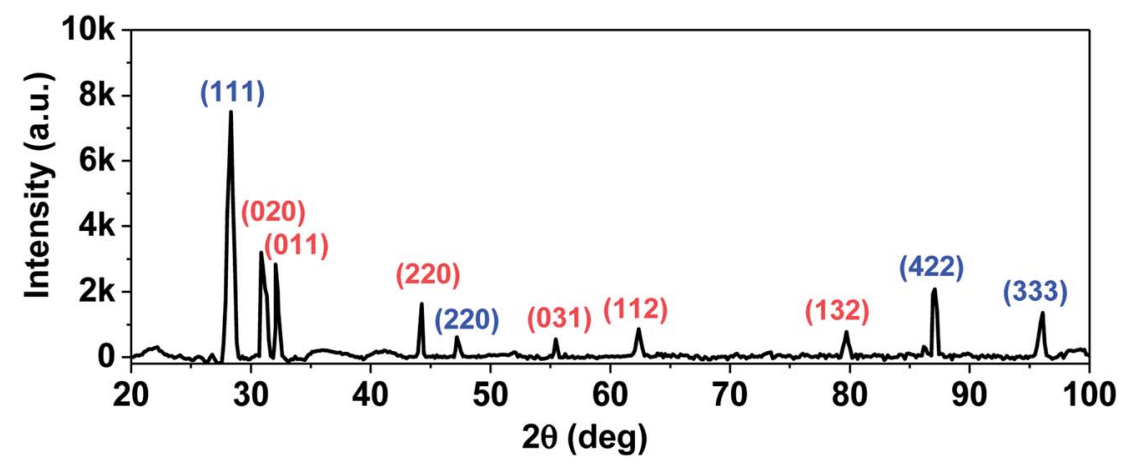

Fig. 8 X-ray Diffraction pattern of Sn-SiNW film. Characteristic peaks that correspond to diffraction planes of Si (blue) and Sn (red) are highlighted.

identified for the lithiation (charging) reactions. These regions correspond to the evolution of a series of lithiated tin and silicon phases beginning with $\mathrm{Li}_{2} \mathrm{Sn}_{5}$ and ending with formation of $\mathrm{Li}_{17} \mathrm{Sn}_{4}$ for the $\mathrm{Sn}$ component of the active mass of the hybrid. In these films, interpreting the SiNW lithiation species as a function of voltage is somewhat more complicated compared with typical voltage profiles of the lithiation of bulk crystalline $\mathrm{Si}$, as there are fewer defined voltage-specific plateaus.

Fig. 9(b) has more of a gradual slope rather than a series of plateaus, as is usual in the case with Sn lithiation voltage profiles. Additionally as the SiNWs are a combination of crystalline and amorphous phases, there will be less transition from crystalline-to-amorphous phase. In Fig. 9(b) the initial lithiation of crystalline bulk silicon results in a low voltage plateau corresponding to a two-phase region in which lithiated amorphous silicon is formed $\left(\mathrm{a}-\mathrm{Li}_{x} \mathrm{Si}\right) \cdot{ }^{54,55}$ Limiting the capacity to around $1000 \mathrm{~mA} \mathrm{~h} \mathrm{~g}^{-1}$ will avoid the deleterious volume expansion, which occurs when the highest lithiated $\mathrm{Si}$ species $\mathrm{Li}_{15} \mathrm{Si}_{4}$ is formed through recrystallisation. This phase formation is associated with an entire amorphous to crystalline transformation, whose onset occurs at around $60 \mathrm{mV}$ and continues when the voltage $<50 \mathrm{mV}$. Such a phase transition can result in high internal stresses, leading to particle fracture and resulting capacity fade - a relationship has been suggested between film delamination and increased $\mathrm{Li}_{15} \mathrm{Si}_{4}$ formation. ${ }^{56}$ This is attributable to the $\mathrm{Li}_{15} \mathrm{Si}_{4}$ crystallization introducing grain boundaries which can in turn cause crack formation to propagate during delithiation, which may result in delamination.

Therefore, if the amorphous phase is maintained by remaining above $50 \mathrm{mV}$ the electrode can in turn retain some structural stability. This is because conventional electrode manufacturing generates electrode architectures (microstructures) that cannot accommodate particle volume expansion to $280 \mathrm{vol} \%$ whilst retaining the cohesive, composite integrity of the as manufactured electrode coating. Obrovac states that the partial lithiation of crystalline Si results in particles composed of lithiated amorphous $\mathrm{Si}$ and completely non-lithiated crystalline $\mathrm{Si}$, as shown schematically at the maximum capacity attained in Fig. 9..$^{54}$

The reversible lithiation characteristics of amorphous silicon have been shown to retain longer structural stability than the crystalline material, attributable to increased tolerance to intrinsic stress and strain. ${ }^{49}$ Specifically the amorphous advantage lies in the ability to facilitate the isotropic stress and strain moments during the lithiation and delithiation processes. $^{49}$

The Sn/Si nanowire hybrid electrode structures (Region ii in Fig. 9a) showed the tin plateaus during the first part of lithiation, and then the silicon lithiation pseudo equilibrium beyond $500 \mathrm{~mA} \mathrm{~h} \mathrm{~g}{ }^{-1}$. The plateau voltage was higher than typically observed for pure silicon electrodes (see Fig. 10). There was a large first cycle loss, and no observable Sn plateaus during delithiation. Part of this was due to the high surface area of the silicon nanowires. However, the absence of the tin voltage features suggests that some of the lithiated tin particles may have become isolated during the silicon expansion and contraction.

The Sn/Si particle electrode (Region iii in Fig. 9a) showed the tin voltage plateaus during delithiation, but not during lithiation, when they were "smeared" out and dominated by the voltage curve where the silicon is undergoing progressive lithiation. The electrode resistance was higher than the nanowire hybrid, due to the reduced number of contact points within the electrode structure. The voltage decreased during the silicon pseudo-equilibrium stage, since the decrease in silicon pseudo OCV was not matched by the usual decrease in resistance.

The discharge capacity as a function of cycle number is shown in 10 and there is a relatively good cycling performance to 100 cycles. However, this electrochemical testing was performed in half-cells against Li foil and this can compensate for the low columbic efficiency (CE) by supplying excessive Li ions as needed (which would not be the case in a full cell that has a finite Li inventory). In commercial cells to achieve industryrelevant cycling performance the CE needs to be $\geq 99.93 \%$ to achieve a target number of charge-discharge cycles (at least 300) before it reaches $80 \%$ capacity retention.

The electrochemical performance of physical vapour deposited (PVD) Sn-Si films was compared with conventional anodes containing powdered (Pwd) Sn-Si hybrid films as outlined in Fig. 11. The anode in (ii) was formulated by conventional means and comprised a polyacrylic acid binder with conductive carbon additives, consistent with conventional electrode manufacturing approaches. The powder hybrid system shows 


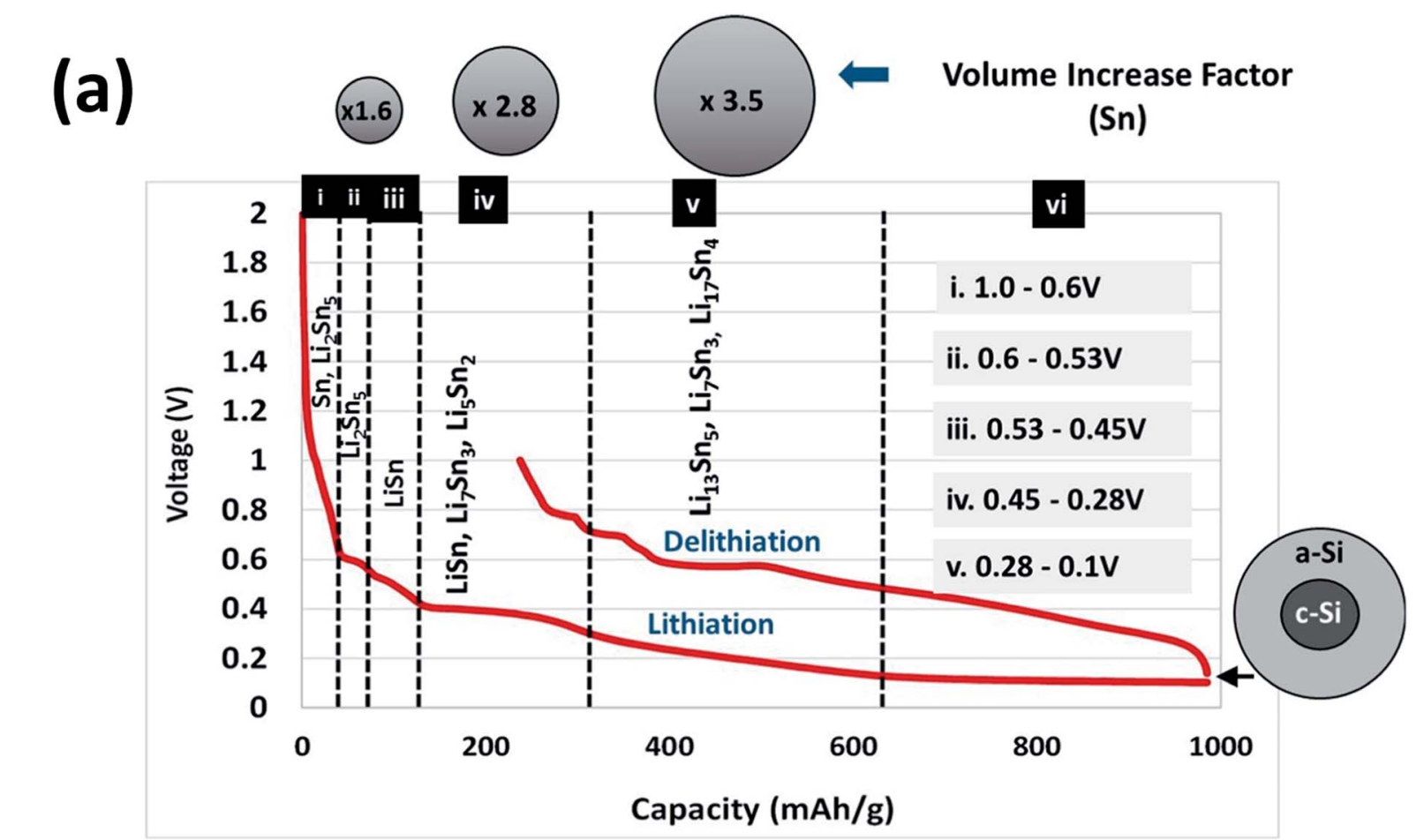

(b)

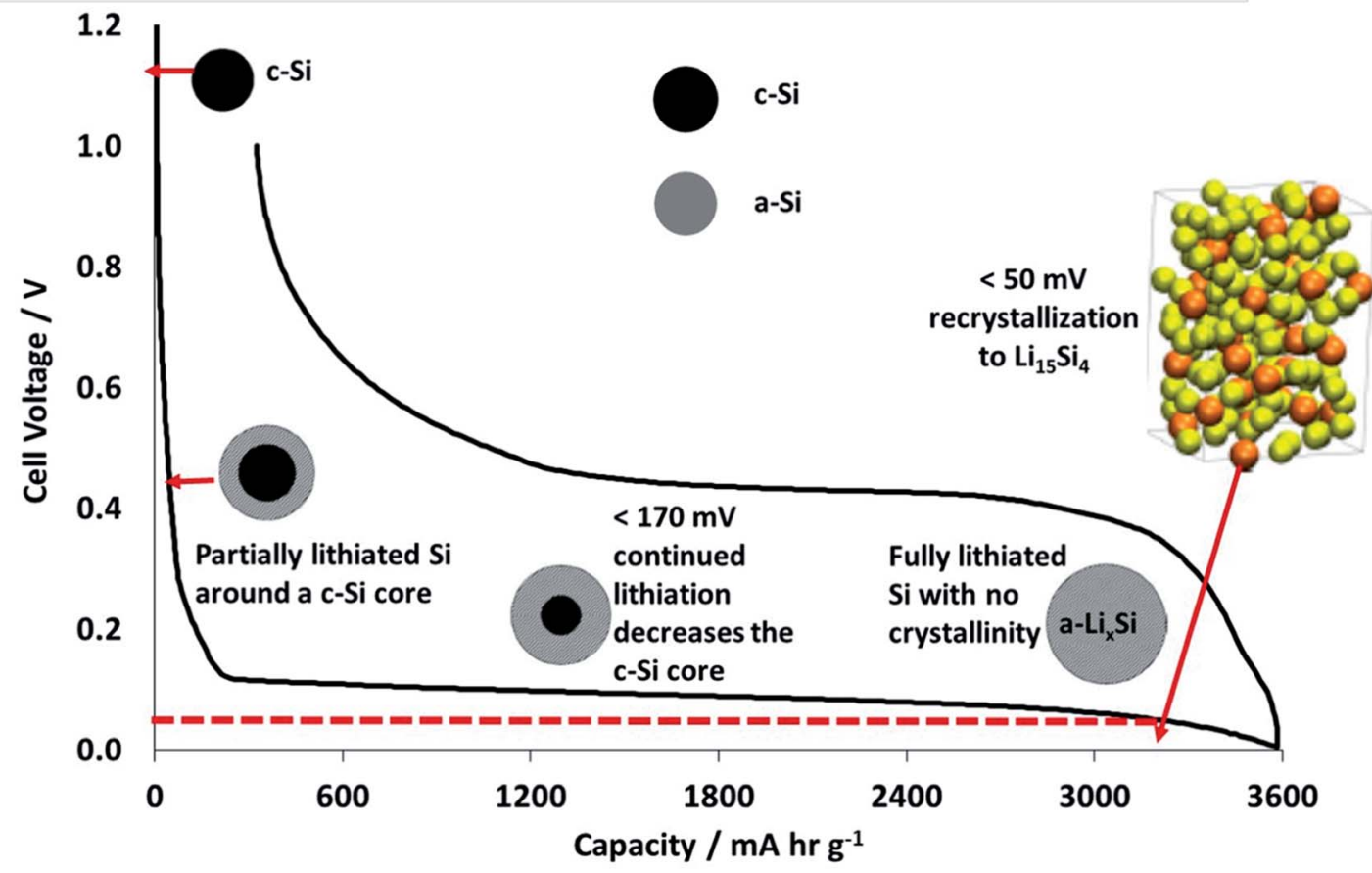

Fig. 9 Voltage-capacity plots for the formation cycle showing key lithiation plateaus and associated voltage ranges (a) SnSi powder hybrid anodes (b) Si powder anodes lithiated to full capacity. NB schematic particles are not intended to be illustrative of scale.

clear accelerated capacity fade rate beyond 80 cycles. The electrode microstructures of the two systems compared are entirely different on several levels, in terms of the electrode film composition and microstructure. Electrodes generated using a conventional composite film deposition approach are often susceptible to delamination phenomena, which result in progressive capacity fade. ${ }^{57,58}$ This can be attributable to the adhesive efficacy of the polymer binder fraction of the composite, often influenced by the polymer's functional groups. ${ }^{59}$ The growth of films of active masses directly from a current collector represents an alternative route to mitigating this effect. This comparative test was carried out to establish whether there is any clear performance benefit to generating a morphologically distinctive, less dense active mass, polymerfree film without the need for conductive additives.

Fig. 12 shows low and high resolution images of VLS-grown SnSi NWs grown on conductive carbon paper, presenting exciting possibilities to generate flexible free-standing 


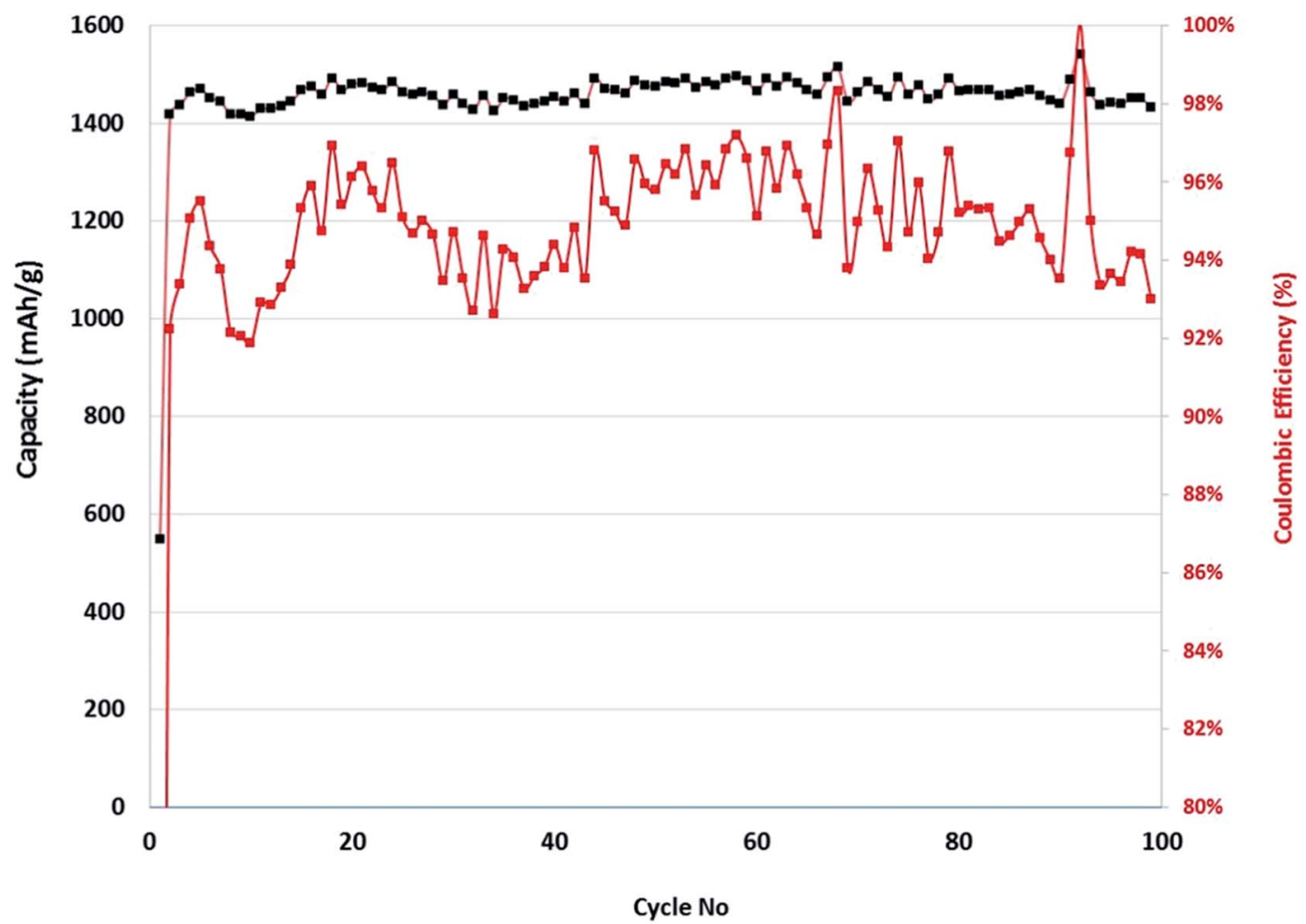

Fig. 10 Discharge capacity \& columbic efficiency vs. cycle number for $\mathrm{Sn}-\mathrm{SiNWs}$ anode vs. Li/Li' .

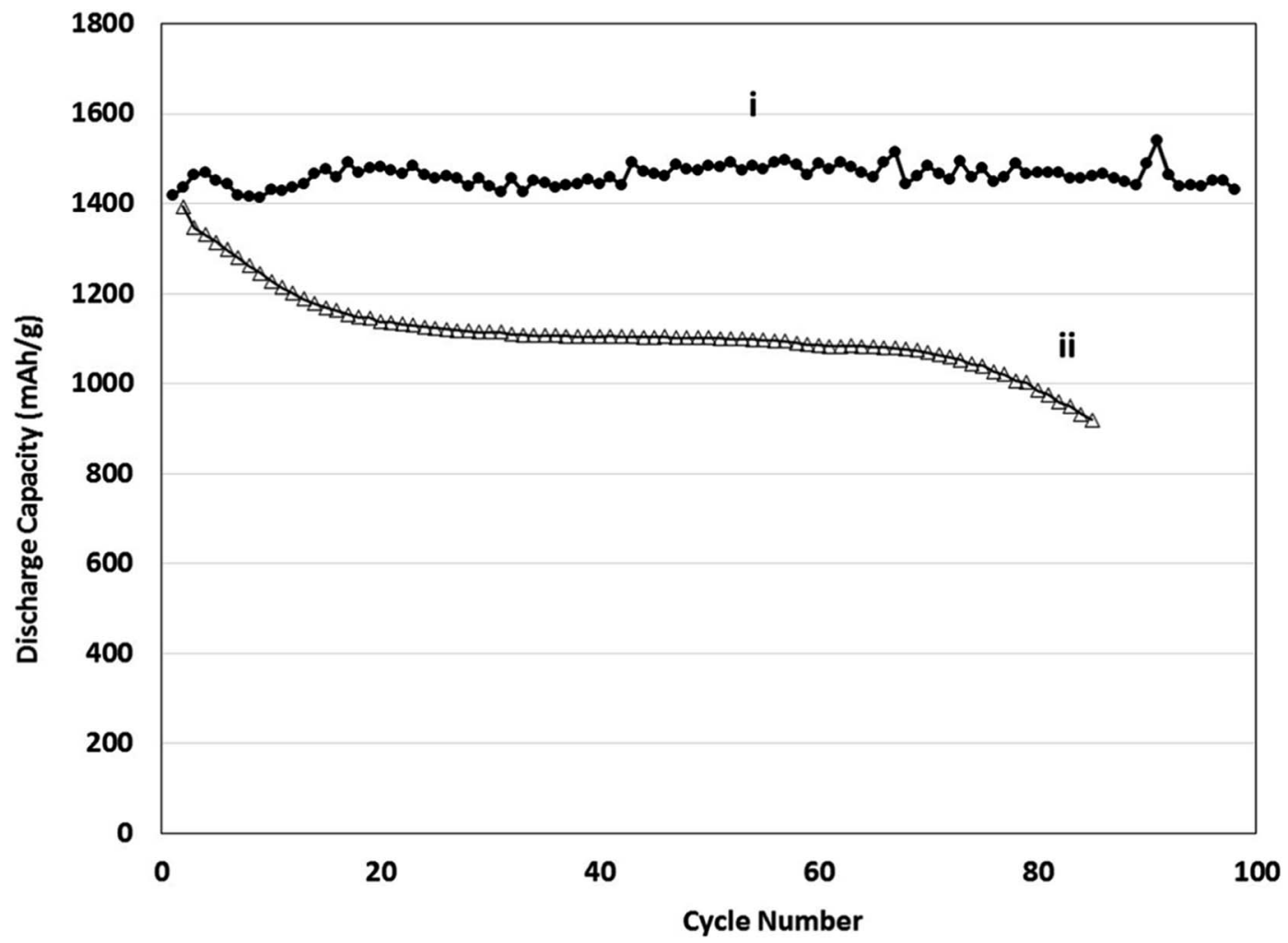

Fig. 11 Discharge capacity vs. cycle number (i) PVD deposited Si-Sn electrodes, (ii) Pwd coated Si-Sn hybrid anodes vs. Li/Li . 


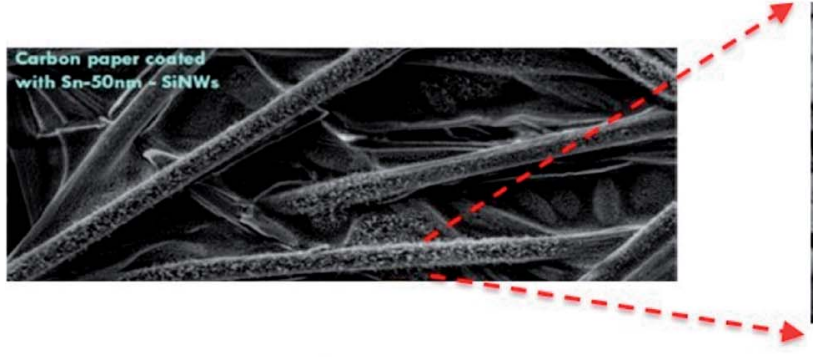

a.

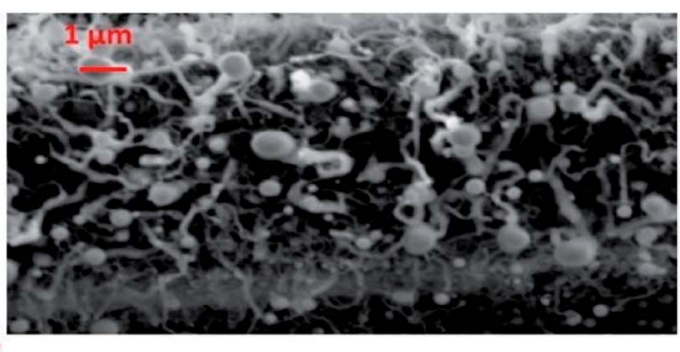

b.

Fig. 12 SEM images of Sn-Si hybrid nanowires grown on carbon cloth at (a) low magnification and (b) higher magnification down to low micron scale.

electrodes incorporating 3D current collector substrates. The electrochemical characterisation of these electrodes will be presented elsewhere in a separate study following the further optimisation of this film growth. $\mathrm{nSi}-\mathrm{C}$ composites have been fabricated by ultra-sonication and positive-pressure filtration ${ }^{60}$ and were shown to stably cycle for a few hundred cycles. The development of flexible electrodes using carbon substrates has the potential to offer excellent thermal, electrical and mechanical properties - especially in applications that require the need to endure cyclical mechanical deformation.

\section{Conclusion}

A relatively low temperature deposition technique was shown to generate a binary anode system composed of both crystalline and amorphous SiNWs, a dense Sn layer and some interfacial copper-tin intermetallic phases. Sn has been shown to be an interesting catalyst for SiNW growth as it is isoelectric with Si thus a neutral "impurity". PECVD of Si in combination with Sn catalyst seeds opens up exciting possibilities at low temperatures. This is facilitated by the dissociation of the precursor gas in a low power plasma, allowing lowering of the substrate temperature whilst maintaining high SiNW growth rate. This non-optimised film was able to endure reversible lithiation for 100 cycles at nearly four times the maximum capacity of graphite. Conventional powder-binder-additive containing electrodes did not achieve this level of performance in a direct cycle life comparison.

Subsequent work will follow on the systematic investigation into optimal SiNW density, morphology, crystallinity, film thickness parameters and alternative $3 \mathrm{D}$ current collectors. Also to be investigated is how to control and optimise deposition parameters on a variety of flexible, conductive substrates. Additional optimisation extending into ternary chemistries may be a possible route to generating more stable amorphous alloy films/metastable structures capable of efficient, reversible lithiation for several hundreds of charge-discharge cycles.

Alternative manufacturing routes such as low temperature PECVD do not require indirect materials or energy-intensive processing, and could be a key enabler generating cheaper and long lasting electrode architectures. Their ability to deposit onto three-dimensional structures could present opportunities beyond the capability of conventional coating techniques. This can enable the use of metallic foam or carbon-based current collectors in batteries for very high rate applications. The potential for use in flexible or wearable energy storage could also become increasingly possible by this approach. Further developing the capability to generate wearable energy storage is of much practical interest although it is far from being at a level ready for commercial exploitation. PECVD is an established, scalable technology that is well-suited to depositing functional semiconductor coatings. Our strategy presents new possibilities to explore and better understand the approaches to designing and understanding new electrode materials and microstructures, to correlate structure with performance, and how to better optimise energy storage components.

\section{Conflicts of interest}

There are no conflicts to declare.

\section{Acknowledgements}

This work was co-funded by EPSRC (Projects AMorpheus and Elevate) and the High Value Manufacturing Catapult at WMG, Warwick University. The research is supported by The Energy Innovation Centre, WMG at Warwick University.

\section{References}

1 C. Daniel, Lithium Ion Batteries and Their Manufacturing Challenges, Bridg.-Front. Eng., 2015, vol. 45, pp. 21-24.

2 A. A. Asif and R. Singh, Further Cost Reduction of Battery Manufacturing, Batteries, 2017, 3(17), 3020017.

3 B. Ludwig, I. Chen and P. H. Pan, Solvent-Free Manufacturing of Electrodes for Lithium-Ion Batteries, in T1B: Lithium-Ion Development \& Commercializattion International Battery Seminar \& Exhibit., Missouri University of Science And Technology, Fort Lauderdale, Florida, 2017.

4 M. Ko, S. Chae and J. Cho, Challenges in Accommodating Volume Change of $\mathrm{Si}$ Anodes for Li-Ion Batteries, ChemElectroChem, 2015, 2, 1645-1651.

5 F. Su, L. Dai, X. Guo, L. Xie, G. Sun and C. Chen, Microstructure evolution and control of lithium-ion battery electrode laminate, Journal of Energy Storage, 2017, 14, 8293, DOI: 10.1016/j.est.2017.09.016.

6 G. A. Elia, U. Ulissi, F. Mueller and J. Reiter, A Long-Life Lithium Ion Battery with Enhanced Electrode/Electrolyte 
Interface by Using an Ionic Liquid Solution, Chem.-Eur. J., 2016, 6808-6814.

7 X. L. Xi, Engineering nanostructured anodes via electrostatic spray deposition for high performance lithium ion, J. Mater. Chem. A, 2012, 1, 164-182.

8 N. J. Dudney, Thin Film Micro-Batteries, Interface, 2008, 4448, available from: https://www.electrochem.org/dl/ interface/fal/fal08/fal08_p44-48.pdf.

9 E. Evshchik, D. Novikov, A. Levchenko, S. Nefedkin and A. V. Shikhovtseva, Magnetron Sputtering Silicon Thin Film Electrodes for Lithium-Ion Batteries, Int. J. Electrochem. Sci., 2018, 13, 2860-2874.

$10 \mathrm{M}$. N. Al-shroofy, Understanding and Improving Manufacturing Processes for Making Lithium-Ion Battery, vol. 3, Theses and Dissertations, Chemical and Materials Engineering 76, University of Kentucky, 2017.

11 H. Tian, F. Xin, X. Wang, W. He and W. Han, ScienceDirect High capacity group-IV elements ( $\mathrm{Si}, \mathrm{Ge}, \mathrm{Sn}$ ) based anodes for lithium-ion batteries, Journal of Materiomics, 2015, 1(3), 153-169.

12 S. Gallanti, M. J. Loveridge and R. Bhagat, Electrodeposition of Si and Sn-based Amorphous Films for High Energy Novel Electrode Materials, MRS Adv., 2017, 2(54), 3249-3254.

13 L. C. Loaiza, E. Salager, N. Louvain, A. Boulaoued, A. Iadecola, P. Johansson, et al., Mechanism of $\mathrm{Si}_{1-x} \mathrm{Ge}_{x}$ alloys, J. Mater. Chem. A, 2017, 5, 12462-12473, DOI: 10.1039/C7TA02100C.

14 E. M. H. White, Novel silicon and tin alloy nano-particulate materials via spark erosion for high performance and high capacity anodes in lithium ion batteries, Graduate Thesis and Dissertations 13081, Iowa State University, 2014.

15 M. Ko, S. Chae and J. Cho, Challenges in Accommodating Volume Change of $\mathrm{Si}$ Anodes for Li-Ion Batteries, ChemElectroChem, 2015, 2, 1645-1651.

$16 \mathrm{H}$. Wu and Y. Cui, Designing nanostructured Si anodes for high energy, Nano Today, 2012, 414-429.

17 J. Xiao, W. Xu, D. Wang, D. Choi, W. Wang, X. Li, et al., Stabilization of Silicon Anode for Li-Ion Batteries, $J$. Electrochem. Soc., 2010, 157(10), A1047, DOI: 10.1149/ 1.3464767.

18 C. Xu, F. Lindgren, B. Philippe, M. Gorgoi, F. Björefors, K. Edström, et al.,Improved Performance of the Silicon Anode for Li-Ion Batteries: Understanding the Surface Modification Mechanism of Fluoroethylene Carbonate as an Effective Electrolyte Additive, Chem. Mater., 2015, 27(7), 2591-2599, DOI: 10.1021/acs.chemmater.5b00339.

19 E. Peled and S. Menkin, Review - SEI: Past, Present and Future, J. Electrochem. Soc., 2017, 164(7).

20 A. Wang, S. Kadam, H. Li, S. Shi and Y. Qi, Review on modeling of the anode solid electrolyte interphase (SEI) for lithium-ion batteries, npj Comput. Mater., 2018, (1-26), DOI: 10.1038/s41524-018-0064-0.

21 M. Zhang, T. Wang, G. Cao, M. Zhang, T. Wang and G. Cao, Promises and challenges of tin-based compounds as anode materials for lithium-ion batteries Promises and challenges of tin-based compounds as anode materials for lithium-ion batteries, Int. Mater. Rev., 2015, 60(6), 330-352.
22 M. N. Obrovac and V. L. Chevrier, Alloy Negative Electrodes for Li-Ion Batteries, Chem. Rev., 2014, 14, 11444-11502.

23 N. Nitta and G. Yushin, High-Capacity Anode Materials for Lithium-Ion Batteries: Choice of Elements and Structures for Active Particles, Part. Part. Syst. Char., 2014, 31, 317-336.

24 M. T. Mcdowell, S. W. Lee, W. D. Nix and Y. Cui, 25th Anniversary Article: Understanding the Lithiation of Silicon and Other Alloying Anodes for Lithium-Ion Batteries, Adv. Mater., 2013, 25, 4966-4985.

25 L. Zhong, C. Beaudette, J. Guo, K. Bozhilov and L. Mangolini, Tin nanoparticles as an effective conductive additive in silicon anodes, Sci. Rep., 2016, 6, 30952.

26 Y. Jin, Y. Tan, X. Hu, B. Zhu, Q. Zheng, Z. Zhang, et al., Scalable Production of the Silicon - Tin Yin-Yang Hybrid Structure with Graphene Coating for High Performance Lithium-Ion Battery Anodes, Electrochim. Acta, 2017, 9, 15388-15393.

27 X. Wang, Z. Wen, Y. Liu and X. Wu, A novel composite containing nanosized silicon and tin as anode material for lithium ion batteries, Electrochim. Acta, 2009, 54, 4662-4667.

28 J. Whiteley, S. Lee, J. M. Whiteley, J. W. Kim, M. Piper and S. Lee, High-Capacity and Highly Reversible Silicon-Tin Hybrid Anode for Solid-State Lithium-Ion Batteries for Solid-State Lithium-Ion Batteries, J. Electrochem. Soc., 2015, 2016, A251-A254.

29 Y. Jin, Y. Tan, X. Hu, B. Zhu, Q. Zheng, Z. Zhang, et al., Scalable Production of the Silicon-Tin Yin-Yang Hybrid Structure with Graphene Coating for High Performance Lithium-Ion Battery Anodes, ACS Appl. Mater. Interfaces, 2017, 9, 15388-15393.

30 C. K. Chan, H. Peng, G. A. O. Liu, K. Mcilwrath, X. F. Zhang, R. A. Huggins, et al., High-performance lithium battery anodes using silicon nanowires, Nat. Nanotechnol., 2008, 3, 31-35.

31 N. Liu, L. Hu, M. T. Mcdowell, A. Jackson and Y. Cui, Prelithiated Silicon Nanowires as an Anode for Lithium Ion Batteries, ACS Nano, 2011, 5(8), 6487-6493.

32 J.-H. Cho and S. T. Picraux, Silicon nanowire degradation and stabilization during lithium cycling by SEI layer formation, Nano Lett., 2014, 14(6), 3088-3095.

33 J. Cho and S. T. Picraux, Enhanced Lithium Ion Battery Cycling of Silicon Nanowire Anodes by Template Growth to Eliminate Silicon Underlayer Islands, Nano Lett., 2013, 13, 5740-5747.

34 I. Ryu, J. Wook, Y. Cui and W. D. Nix, Size-dependent fracture of Si nanowire battery anodes, J. Mech. Phys. Solids, 2011, 59(9), 1717-1730, DOI: 10.1016/ j.jmps.2011.06.003.

35 B. A. Korgel, Twins cause kinks, Nano Lett., 2006, 5, 521-522. 36 K. Peng, X. Wang, S. Lee, K. Peng, X. Wang and S. Lee, Silicon nanowire array photoelectrochemical solar cells Silicon nanowire array photoelectrochemical solar cells, Appl. Phys. Lett., 2008, 163103, 1-4.

37 H. Choi, Vapor-Liquid-Solid Growth of Semiconductor, ed. Y. G. C. First, Springer, Berlin, 2012, pp. 1-36.

38 R. S. Rana, R. Purohit and S. Das, Reviews on the Influences of Alloying elements on the Microstructure and Mechanical 
Properties of Aluminum Alloys and Aluminum Alloy Composites, Int. J. Sci. Res. Publ., 2012, 2(6), 1-7.

39 V. B. Neimash and A. O. Goushcha, Self-sustained cyclic tin induced crystallization of amorphous silicon, J. Mater. Res., 2015, 30(20), 3116-3124.

40 R. W. Olesinski and G. J. Abbaschian, Numerical Data and Functional Relationships, in Science and Technology, Springer, Berlin, Heidelberg, 1984, vol. 5.

41 R. E. Smallman and R. J. Bishop, Modern Physical Metallurgy \& Materials Engineering, Butterworth Heinmann, 6th edn, 1999, p. 48.

42 J. Tang, J. Maurice, W. Chen, S. Misra, M. Foldyna and E. V. Johnson, Plasma-Assisted Growth of Silicon Nanowires by Sn Catalyst: Step-by-Step Observation, Nanoscale Res. Lett., 2016, 11, 455.

43 Y. Kuang, V. M. Di, J. K. Rath, L. Yu, B. O. Donnell, M. Foldyna, et al., A review on plasma-assisted VLS synthesis of silicon nanowires and radial junction solar cells, J. Phys. D: Appl. Phys., 2014, 47, 393001.

44 L. Sun, Y. Fan and X. Wang, Vapor-Liquid-Solid Growth of Small- and Uniform-Diameter Silicon Nanowires at Low Temperature from $\mathrm{Si}_{2} \mathrm{H}_{6}$, Appl. Phys. Express, 2008, 1, 14003.

45 S. J. Rathi, B. N. Jariwala, J. D. Beach, P. Stradins, P. C. Taylor, X. Weng, et al., Tin-Catalyzed Plasma-Assisted Growth of Silicon Nanowires, J. Phys. Chem. C, 2011, 115, 3833-3839.

46 H. Zhang, J. Yang, H. Hou, S. Chen and H. Yao, Nitrogendoped carbon paper with $3 \mathrm{D}$ porous structure as a flexible free-standing anode for lithium-ion batteries, Sci. Rep., 2017, 7(7769), 1-9.

47 M. Pritchett and J. A. Kelber, Adherence/Diffusion Barrier Layers for Copper Metallization, Amorphous Carbon:Silicon Polymerized Films, PhD thesis, University of North Texas, 2004.

48 B. Tian, P. Xie, T. J. Kempa, D. C. Bell and C. M. Lieber, Single-crystalline kinked semiconductor nanowire superstructures, Nat. Nanotechnol., 2009, 4(12), 824-829.

49 L. Lin, X. Xu, C. Chu, M. K. Majeed and J. Yang, Mesoporous Amorphous Silicon: A Simple Synthesis of a High-Rate and Long-Life Anode Material for Lithium-Ion Batteries, Angew. Chem., Int. Ed., 2016, 55, 14063-14066.

50 M. N. Obrovac and L. Christensen, Structural Changes in Silicon Anodes during Lithium Insertion/Extraction,
Electrochem. Solid-State Lett., 2004, 7(5), A93, DOI: 10.1149/ 1.1652421.

51 K. Ogata, E. Salager, C. J. Kerr, A. E. Fraser, C. Ducati, A. J. Morris, et al., Revealing lithium-silicide phase transformations in nano-structured silicon-based lithium ion batteries via in situ NMR spectroscopy, Nat. Commun., $2014,5,3217$.

52 J. Wu, Z. Zhu, H. Zhang, H. Fu, H. Li and A. Wang, A novel Si/ Sn composite with entangled ribbon structure as anode materials for lithium ion battery, Sci. Rep., 2014, 6(29356), $1-8$.

53 S. Y. Shiryaev, J. L. Hansen, P. Kringho, A. N. Larsen, S. Y. Shiryaev, J. L. Hansen, et al., Pseudomorphic $\mathrm{Si}_{1-x} \mathrm{Sn}_{x}$ alloy films grown by molecular beam epitaxy on Si, Appl. Phys. Lett., 1995, 16, 2287.

54 M. N. Obrovac and L. J. Krause, Reversible Cycling of Crystalline Silicon Powder, J. Electrochem. Soc., 2007, 154(2), A103, DOI: 10.1149/1.2402112.

55 M. J. Loveridge, M. J. Lain, Q. Huang and C. Wan, Enhancing cycling durability of Li-ion batteries with hierarchical structured silicon - graphene, Phys. Chem. Chem. Phys., 2016, 18(i), 30677-30685.

56 D. Iaboni, $\mathrm{Li}_{15} \mathrm{Si}_{4}$ Formation in Thin Film $\mathrm{Si}$ Negative Electrodes for Li-ion Batteries, MSc dissertation, Dalhousie University, Dalhousie, 2015.

57 M. Baunach, S. Jaiser, S. Schmelzle, H. Nirschl, P. Scharfer, W. Schabel, et al., Delamination behavior of lithium-ion battery anodes: Influence of drying temperature during electrode processing, Drying Technol., 2016, 34(4), 462-473, DOI: 10.1080/07373937.2015.1060497.

58 B. Son, M. Ryou, J. Choi, T. Lee, H. K. Yu, J. H. Kim, et al., Measurement and Analysis of Adhesion Property of Lithium-Ion Battery Electrodes with SAICAS, ACS Appl. Mater. Interfaces, 2014, 6, 526-531.

59 S.-L. Chou, Y. Pan, J.-Z. Wang, H.-K. Liu and S.-X. Dou, Small things make a big difference: binder effects on the performance of $\mathrm{Li}$ and $\mathrm{Na}$ batteries, Phys. Chem. Chem. Phys., 2014, 16(38), 20347-20359.

60 J. P. Zheng, Binder-free freestanding flexible Si nanoparticle - multi-walled carbon nanotube composite paper anodes for high energy Li-ion batteries, J. Mater. Res., 2018, 482-494. 